\title{
421 - Prevalence of Pain in Dementia Subtypes: Data from a National Sample of Aged Care Residents in Australia
}

Atee $\mathrm{M}_{1}{ }^{1,2}$ Morris $\mathrm{T}^{3}$ Macfarlane $\mathrm{S}^{3,4}$ Cunningham $\mathrm{C}^{3,5}$

${ }_{1}^{1}$ The Dementia Centre, HammondCare, Wembley, WA, Australia

${ }^{2}$ Curtin Medical School, Faculty of Health Sciences, Curtin University, Bentley, WA, Australia

${ }^{3}$ The Dementia Centre, HammondCare, St Leonards, NSW, Australia

${ }^{4}$ Faculty of Medicine, Nursing \& Health Sciences, Monash University, Clayton, VIC, Australia

${ }^{5}$ School of Public Health \& Community Medicine, University of New South Wales, Sydney, NSW, Australia

Email: matee@dementia.com.au

Background: Pain is poorly identified in dementia due to complete or partial loss in communication, which is associated with progressive cognitive impairment. If it goes untreated, pain can lead to behavioral disturbances (e.g., agitation/aggression), delirium, inappropriate pharmacotherapy (e.g., psychotropics), hospitalizations and caregiver distress. There are limited prevalence data in the literature on pain in dementia subtypes.

Objective: This study aims to investigate the prevalence and intensity of pain in various dementia subtypes in aged care residents living with dementia (RLWD), using a technology-driven pain assessment tool.

Methods: A 1-year retrospective cross-sectional study was conducted on the presence and intensity of pain in referrals to Dementia Support Australia from residential aged care homes (RACHs), using PainChek ${ }^{\circ}$. PainChek ${ }^{\circ}$ is a pain assessment tool that uses artificial intelligence algorithms (e.g., automated facial recognition and analysis) to identify facial expressions indicative of pain in conjunction with other digital checklists of pain behaviors such as vocalization and movement cues. Presence and intensity of pain were identified using PainChek ${ }^{\circ}$ categories (scores): no pain (0-6), mild pain (7-11), moderate pain (12-15) and severe pain (16-42).

Results: During the study period (01/11/2017-31/10/2018), a sample of 479 referrals (age: $81.9 \pm 8.3$ years old; $55.5 \%$ female) from 370 RACHs with Alzheimer's disease (AD; 40.9\%), vascular dementia (VaD; $12.7 \%$ ), mixed dementia (MD; 5.9\%), dementia with Lewy body (DLB; $2.9 \%$ ), and frontotemporal dementia (FTD; 2.3\%) were examined. Pain was prevalent in two-thirds $(65.6 \%)$ of the referrals with almost half $(48.4 \%)$ of these categorized as experiencing moderate-severe pain. MD and those with DLB (78.6\% each) shared the highest prevalence of pain, followed by $A D(64.3 \%)>\operatorname{VaD}(62.3 \%)>$ FTD (54.6\%). Prevalence of severe pain was as follow: $\operatorname{MD}(17.9 \%)>\operatorname{AD}(12.3 \%)>\operatorname{VaD}(11.5 \%)>$ FTD $(9.1 \%)>$ DLB (7.1\%).

Conclusion: To date, this is the largest study that presented data on pain prevalence and intensity in major dementia subtypes in the RACH setting. Moderate-severe pain is highly prevalent in RLWD, which appears to differ by dementia subtypes. This may reveal the impact of neuropathological etiology of those subtypes on the neurobiology of pain.

Word count: 344 words

Atee M, Morris T, Macfarlane S, Cunningham C. Pain in Dementia: Prevalence and Association with Neuropsychiatric Behaviors. J Pain Symptom Manage. Oct 14 2020;61(6):1216-1227.

doi:10.1016/j.jpainsymman.2020.10.011 